Recepción: 10/ 08 / 2018

Aceptación: 22 / 10 / 2018

Publicación: 03 / 12 / 2018

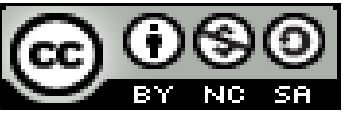

Ciencias de la salud

Artículo de revisión

\title{
Caracterización de la gestión de la seguridad y salud laboral en el Ecuador
} desde la perspectiva de la investigación científico-académica

\section{Characterization of occupational health and safety management in Ecuador from the perspective of scientific-academic research}

Caracterização da gestão da saúde ocupacional e segurança no Equador sob a perspectiva da pesquisa científico-acadêmica

\author{
Tomas V. Otero-Gorotiza ${ }^{\mathrm{I}}$ \\ totero2@hotmail.com \\ Robín A. Torres-Bravo II \\ oejtb2011@hotmail.com \\ Wenceslao A. Mite-Calero III \\ wences05@yahoo \\ Luis A. Anchundia-Santana IV \\ assiul_2112@hotmail.com
}

Correspondencia: totero2@hotmail.com

\footnotetext{
I Magíster en Seguridad, Salud y Ambiente, Ingeniero Industrial, Docente de la Universidad Central del Ecuador, Quito, Ecuador.

II Magíster en Seguridad, Salud y Ambiente, Ingeniero Industrial, Docente de la Universidad Central del Ecuador, Quito, Ecuador.

III Magíster en Seguridad y Salud y Ambiente, Psicólogo Industrial, Docente de la Universidad Central del Ecuador, Quito, Ecuador.

IV Magíster en Seguridad y Salud y Ambiente, Ingeniero Industrial, Docente de la Universidad Central del Ecuador, Quito, Ecuador.
} 


\section{Resumen}

La gestión de la seguridad y la salud laboral es hoy en día un pilar fundamental para las organizaciones en su búsqueda de mejores condiciones de trabajo como base para alcanzar mayor productividad y rentabilidad. Las iniciativas de investigación deberían estar en sintonía con la problemática global, y la academia debería ser la tribuna para la discusión y divulgación de las tendencias en gestión de seguridad y salud laboral. El objetivo de esta investigación es caracterizar la gestión de la seguridad y salud laboral en el contexto ecuatoriano desde una perspectiva de los actores científico-académica. A partir de una revisión documental, se presenta un análisis y discusión, con unas reflexiones finales.

Palabras clave: seguridad; salud laboral; productividad.

\section{Abstract}

Occupational health and safety management is today a fundamental pillar for organizations in their search for better working conditions as a basis to achieve greater productivity and profitability. The research initiatives should be in line with the global problem, and the academy should be the platform for the discussion and dissemination of trends in occupational health and safety management. The objective of this research is to characterize the management of occupational health and safety in the Ecuadorian context from a scientific-academic perspective. From a documentary review, an analysis and discussion is presented, with some final reflections.

Key words: security; occupational health; productivity.

\section{Resumo}

A gestão da saúde ocupacional e segurança é hoje um pilar fundamental para as organizações em busca de melhores condições de trabalho como base para alcançar maior produtividade e rentabilidade. As iniciativas de pesquisa devem estar alinhadas com o problema global, e a academia deve ser a plataforma para a discussão e disseminação de tendências na gestão da saúde ocupacional e segurança. O objetivo desta pesquisa é caracterizar a gestão da saúde e segurança ocupacional no contexto equatoriano a partir de uma perspectiva científico-acadêmica. A partir de uma revisão documental, é apresentada uma análise e discussão, com algumas reflexões finais.

Palavras chave: segurança; saúde ocupacional; produtividade. 


\section{Introducción}

La gestión de la seguridad y de la salud en el trabajo, se viene consolidando a nivel mundial, como un pilar necesario de toda organización para mejorar las condiciones de sus trabajadores alcanzando una mayor productividad $\mathrm{y}$, por consiguiente, una mayor rentabilidad de sus actividades laborales (Arias-Mendoza, 2017). La seguridad y la salud en el trabajo no había sido en el pasado, objeto de preocupación de todas las partes interesadas en el contexto organizacional, sin embargo, hoy en día, se está convirtiendo en el tema clave de las preocupaciones globales (Jilchaa \& Kitaw, 2017).

Es primordial mantener una gestión eficiente y continua en el sistema de seguridad y salud ocupacional, porque permite garantizar la calidad de vida de trabajador y de la organización (Jiménez, 2017).

La protección de la salud y seguridad de los trabajadores es un proceso complejo que requiere de la participación de todos los integrantes de la cadena de valor de una organización con el fin de realizar intervenciones sistémicas y multidisciplinarias que logren dar respuesta a los problemas que aquejan a los grupos laborales desde la perspectiva del trabajo prescrito y del trabajo real (Rodríguez, 2010). La moderna concepción de la seguridad y salud en el trabajo se diferencia de sus precedentes en la enorme amplitud de sus objetivos y en la gran ambición de sus instrumentos (Romeral, 2012).

Los riesgos en la vida y la salud de las personas han existido desde siempre, por lo que han tenido la necesidad de protegerse; cuando se presentan estos hechos en el ámbito laboral, históricamente, solo el factor de la producción ha tenido más énfasis que el de la salud y seguridad, debido a que recientemente las personas han tomado conciencia de la importancia que tiene estos factores en el trabajo (Heredia, Benítez \& Marcillo, 2017).

Una empresa que no asuma a la seguridad y salud ocupacional, como un elemento importante en la institución, y no destine los recursos necesarios para este aspecto, estará en un escenario de riesgo alto, que en mucho de los casos le puede conducir a su quiebra financiera (Jiménez, 2017). Así, un sistema de gestión de seguridad y salud en el trabajo es parte integrante del sistema de gestión de una empresa u organización, empleado para desarrollar e implementar su política en la 
materia y gestionar sus riesgos para la seguridad y salud en el trabajo (Luna, Álvarez \& Soledispa, 2017).

La salud ocupacional y la seguridad industrial conforman un binomio que no pueden ser separados debido a que garantizan la minimización de los riesgos de trabajo y la prevención de accidentes para que exista un adecuado clima laboral en la organización y se pueda generar mayor productividad en el desarrollo de las actividades (Heredia, Benítez \& Marcillo, 2017).

Como lo plantean Carvajal \& Pellicer (2009), a pesar de que, la seguridad y la salud en el trabajo han sufrido cambios significativos durante los últimos años, producto de la presión ejercida por los trabajadores y por la sociedad a causa del gran número de accidentes laborales, la investigación en este campo es poco desarrollada lo que da a entender que desde este ámbito se puede hacer un gran aporte a la creación de una verdadera cultura de la seguridad, mediante la formación e información adecuada a todos los agentes participantes.

El objetivo de esta investigación es caracterizar la gestión de la seguridad y salud laboral en el contexto ecuatoriano, desde la perspectiva de la investigación científico-académica. El documento está estructurado partiendo de la introducción y presentación de la metodología; los resultados se presentan describiendo la seguridad y salud laboral como gestión, su quehacer en el ambiente de investigación, su dinámica en la academia, su presencia en el contexto ecuatoriano, y las reflexiones finales.

\section{Metodología}

Para este documento se ha hecho una selección de los artículos sobre seguridad y salud ocupacional publicados con enfoque de gestión en los recursos Google Académico®, ScienceDirect®, entre otros. Se usaron como palabras clave, seguridad y salud ocupacional, seguridad y salud laboral, y Ecuador, para identificar referentes en el contexto ecuatoriano.

Aunque el enfoque de la investigación es de carácter descriptiva, se apoya en la revisión documental. Se analizan los resultados y se reflexiona de la información obtenida, bajo la perspectiva académico-investigativa. 


\section{Seguridad y salud laboral desde la investigación y la academia}

Los riesgos para la seguridad y la salud en el lugar de trabajo hoy en día se consideran una fuerza impulsora para buscar soluciones para evitar que los empleados de las industrias manufactureras tengan consecuencias negativas; en los últimos años, los requisitos de calidad, salud, conocimiento y seguridad en muchos países han sido más estrictos de lo que se vio anteriormente (Jilchaa \& Kitaw, 2016).

Para Rodríguez, 2010), la protección de la salud y seguridad de los trabajadores se logra a través de tres grandes áreas de conocimiento que, de manera entrelazada, permiten adecuar el medio ambiente y los métodos de trabajo a las capacidades de los individuos: ergonomía, higiene y seguridad industrial.

La seguridad y salud laboral (SSL), o seguridad y salud ocupacional (SSO) es un área multidisciplinaria preocupada principalmente por la salud, seguridad y bienestar de los trabajadores; que, además, debería ocuparse de la protección entre otros a las familias, clientes, proveedores de las cadenas de suministros y comunidades vecinas que pudieran ser impactadas por accidentes (Rodríguez \& Torre, 2010). Es así como, en las últimas décadas, el estudio de la enfermedad profesional y del accidente de trabajo ha ido cediendo paso al más amplio y ambicioso de la promoción de la mejora de la salud y el bienestar en el trabajo (Romeral, 2012).

Con la aparición de Internet, empezó a manejarse mayor cantidad de datos e información a una gran velocidad, lo que ha permitido ver a la seguridad y salud laboral como producto especializado para los profesionales de toda índole como ingenieros, consultores, médicos, investigadores, administradores, comunicadores sociales, filósofos y psicólogos (Rodríguez \& Torre, 2010).

Desde finales del siglo $\mathrm{XX}$, las condiciones de trabajo han venido variando sustancialmente, producto de factores como la innovación tecnológica, la flexibilidad en el empleo, la incorporación de la mujer, la diversidad, la internacionalización de las empresas, las fusiones, la subcontratación, entre otros; estos nuevos factores han incrementado los riesgos para la seguridad y salud de los trabajadores, incluyendo, los traslados al lugar de trabajo y los factores de riesgo 
psicosocial, que provienen del ritmo, logro de metas y el aprendizaje continuo en un estado de competencia constante como uno de los principales estresores (Martínez \& Yandún, 2017).

El nuevo enfoque de la gestión de la seguridad y salud laboral, trasciende la simple evitación o reducción de los riesgos para abarcar una política global de mejora de las condiciones de trabajo en que se desenvuelve el trabajador, basada en la visión unitaria de la empresa, donde quede integrada: la planificación, la coordinación, la participación, el control de la eficacia, la información y la formación (Romeral, 2012).

En cuanto al componente investigativo, el mayor esfuerzo sobre accidentes y su prevención con enfoque de gestión, es producido por las universidades de Estados Unidos y Canadá, publicado en idioma inglés, con una alta preferencia de los artículos de revista por sobre las conferencias (Rodríguez \& Torre, 2010).

Actualmente, los centros de investigación en esta materia reportan la necesidad de incluir en el análisis aspectos tales como organización del trabajo, costo cognitivo de las tareas, fiabilidad y resiliencia, dado el número creciente de estudios que han podido demostrar la relación de estos factores y el desencadenamiento de Trastornos Musculo Esqueléticos y accidentes de trabajo, entre otros (Rodríguez, 2010).

En estudios realizados por Carvajal \& Pellicer (2009), los autores concluyen que el área de evaluación de riesgos es la que cuenta con mayor número de publicaciones; así mismo, las áreas de prevención de riesgos y análisis de accidentes están prácticamente al mismo nivel en cuanto a investigación, ya que cuentan con porcentajes muy similares de publicaciones. De acuerdo con Romeral (2012), la evaluación de riesgos es una actividad que debe ser realizada por personal debidamente cualificado y que tenga la formación legalmente requerida, bien sean trabajadores designados por la dirección de la empresa o formen parte del servicio de prevención propio o ajeno; sin embargo, su realización es responsabilidad de la gerencia de la empresa, aunque debe consultarse a los trabajadores o a sus representantes sobre el método empleado para realizarla, y hay que tener en cuenta que el método deberá ajustarse a los riesgos existentes y al nivel de profundización requerido. 
En cuanto al marco normativo y reglamentario, Carvajal \& Pellicer (2009), sostienen que se cuenta con muy poca bibliografía, a pesar de existir a nivel mundial una normativa avanzada en seguridad y salud, y constituir un instrumento indispensable para desarrollar una cultura en seguridad y salud en la construcción. Agregan estos autores que, el área reglamentaria, es, por lo tanto, otra área que requiere ser investigada teniendo en cuenta aspectos como la unificación de criterios y clarificación de términos.

En este sentido Romeral (2012) plantea que, existen dos vertientes legislativas en el tratamiento de la seguridad y salud laboral, una desde el punto de vista reparador o de protección del trabajador cuando el daño se ha materializado, y otra desde el punto de vista preventivo, actuando antes de que el daño se materialice; es decir, en el origen del riesgo, de forma que se evite su materialización, y con ello la puesta en marcha de la vía resarcitoria.

Jilchaa \& Kitaw (2016), plantean que las tendencias en el control de seguridad y salud en el lugar de trabajo, carecen de estudios de sistemas de gestión integrados y universales. En este sentido, Luna, Álvarez \& Soledispa (2017) señalan que, un Sistema de Gestión de Seguridad y Salud en el Trabajo, contempla la identificación y evaluación de los factores de riesgos laborales a fin de particularizar la aplicación de medidas de control sobre los mismos, con el fin de eliminar o minimizar las consecuencias negativas que pudieran ocurrir sobre el trabajador y el medio ambiente laboral; y que, adicionalmente, involucra un esfuerzo conjunto del personal que conforma la organización en tema de prevención de manera que los derechos y responsabilidades sean compartidos en lo referente al tema de seguridad y salud en el trabajo.

En cuanto a la actividad de investigación en seguridad y salud laboral, Jilchaa \& Kitaw (2016), señalan que esta actividad presenta brechas que requieren áreas de investigación ampliadas, destacando la diferencia de cultura de seguridad que afecta el lugar de trabajo, las metodologías de investigación con variaciones que no son holísticas, la medición de desempeño de inestabilidad de los enfoques en el lugar de trabajo, el dinamismo de la innovación tecnológica y su impacto en la seguridad en el lugar de trabajo, la ausencia de distinción clara entre sistemas de gestión de la seguridad en países desarrollados y en desarrollo, la ausencia de investigaciones multidisciplinarias y falta de impacto en la integración del sistema de gestión. 
Rodríguez \& Torre (2010), señalan que las investigaciones realizadas en seguridad y salud laboral están orientados mayormente a la prevención de accidentes y tienen una orientación operacional enfocada a procedimientos preventivos y normativos, identificación de riesgos y seguimiento de los mismos a través del diseño de indicadores de gestión; agregan que , estos trabajos tienen poca orientación hacia la planificación estratégica sostenible a largo plazo que requieren el desarrollo de programas de mejora continua del sistema de seguridad y salud laboral y su integración con los sistemas de calidad y gestión ambiental.

La identificación y evaluación de los peligros y riesgos de salud y seguridad en el trabajo y sus impactos, facilitan la integración al sistema de gestión de la calidad y permiten cubrir las no conformidades relacionadas con el ambiente de trabajo que mantiene la norma ISO 9001 (UlloaEnríquez, 2012).

En el marco del desarrollo sostenible, los grupos de salud y de seguridad en su lugar de trabajo representan un problema para las organizaciones, por lo que el desarrollo de entornos sostenibles de seguridad y salud ocupacional se han convertido en una prioridad global (Jilchaa \& Kitaw, 2017).

Al enfrentar estos desafíos en la gestión de seguridad y salud laboral, debe plantearse, tal como lo sostienen Jilchaa \& Kitaw (2017), el papel que la innovación en seguridad y salud en el lugar de trabajo produce en el desarrollo sostenible de los ciudadanos y como avanza por los caminos hacia un desarrollo sostenible en la salud industrial de la mano de obra. Agregan Jilchaa \& Kitaw (2017) que, el dinamismo del desarrollo socioeconómico ha traído cambios en todos los aspectos de la expansión, y que este cambio dinámico en la innovación de salud y seguridad en el lugar de trabajo, la alta prevalencia de enfermedades ocupacionales y el registro de accidentes son los factores iniciales del desarrollo sostenible, por lo que, la existencia de problemas de seguridad y salud ocupacional está impactando el nivel de pobreza a la carga existente de la sociedad.

En los actuales momentos, la esfera mental dentro de un análisis macro ergonómico, es el de mayor incidencia, para lo que es necesario crear estrategias que permitan reducir estos factores, porque conduce a la generación de una de las enfermedades más frecuente como es el estrés (Jiménez, 2017). En el curso de las investigaciones futuras, los cambios políticos, sociales, 
demográficos y climáticos serán determinantes; por lo que, la identificación de conexiones entre los cambios organizacionales y la salud mental, Trastornos Musculo Esqueléticos, y otras manifestaciones tempranas de patologías asociadas al trabajo se constituyen como oportunidades de indagación científica pertinente y con un carácter profundamente social (Rodríguez, 2010).

El gasto que se destina en prevenir y mantener la salud del trabajador se la debe plantear como una inversión, porque con base en este rubro se puede lograr mejoras en la productividad y por ende en la rentabilidad (Jiménez, 2017).

Otro aspecto importante en la investigación en seguridad y salud laboral, como lo reportan Rodríguez \& Torre (2010), es el flujo de trabajos de investigación entre los países desarrollados, donde el 35\% de las investigaciones en este campo migra o se exporta para publicarse en una región diferente a la que se produce; en este panorama, Europa es el centro principal de publicación con $63 \%$ de los trabajos, debido mayormente a las importaciones de publicaciones de Estados Unidos, Canadá, China y Australia.

\section{El contexto ecuatoriano}

El Ecuador, considerando los lineamientos mundiales con base en la protección del trabajador, se encuentra en un período de transición y solidificación de sus leyes sobre seguridad y salud en el trabajo (Arias-Mendoza, 2017). La cantidad de material referente a seguridad y salud laboral en el contexto ecuatoriano no es escasa; sin embargo, considerando el contexto global, necesita de mayor interés por parte de la comunidad científico-académica.

De acuerdo con el artículo 326 de la Constitución de la República del Ecuador (2008), en el numeral 5, "Toda persona tendrá derecho a desarrollar sus labores en un ambiente adecuado y propicio, que garantice su salud, integridad, seguridad, higiene y bienestar”.

La gestión de la seguridad y salud en el trabajo y la implantación de su sistema, se han convertido en nuevo tema en el Ecuador, aunque legalmente no lo sean; transformándose, de ser un requisito de obligatorio cumplimiento por las organizaciones, a un modelo de permanencia y eficiencia productiva en las mismas (Arias-Mendoza, 2017).

Tras la realización de la comparación realizada por Martínez \& Yandún (2017), se evidencia que la legislación ecuatoriana mediante los cuerpos legales existentes cumple con lo manifestado por 
ISO 26000 que es de carácter voluntario; es decir, que las organizaciones ecuatorianas que cumplen con la parte legal en seguridad y salud laboral, cumplen con los principios enmarcados en la norma ISO 26000 de responsabilidad social. Este aspecto constituye una buena plataforma para consolidar iniciativas en este sentido.

De acuerdo con Gómez, et al. (2018), diversos estudios han estimado los accidentes de trabajo y las enfermedades profesionales desde un enfoque por exposición a factores de riesgo laboral en diferentes países, planteando metodologías para establecer estimaciones sobre los accidentes de trabajo, debido a que no se disponen de estadísticas fiables; para en el caso de Ecuador, a pesar del incremento considerable del número de casos notificados por accidentes de trabajo en los últimos años (tasa de incidencia x 100.000 trabajadores; 381,2 en 2010 a 775,0 en 2015), igualmente se evidencia la existencia de un subregistro de accidentes de trabajo en el país.

Gómez, et al. (2018), Se encuentran en la ciudad de Quito una aproximación de la siniestralidad laboral declarada por los trabajadores donde pueden ayudar a formular hipótesis sobre los factores de riesgo laboral que pudieran intervenir en la cadena causal de los accidentes de trabajo y la formulación de un nuevo un marco normativo adaptado al contexto ecuatoriano que permitan establecer un plan nacional de seguridad y salud en el trabajo.

Por su parte, Gómez \& Suasnavas (2015), establecen una aproximación sobre la siniestralidad laboral en Ecuador y, a su vez, la comparación entre países. Entre los resultados más interesantes se destaca que, las notificaciones de accidentes de trabajo registrados en 2012 representan una incidencia de 550,53 casos por cada 100.000 trabajadores, índice superior al registrado en 2011, con 419,76. En 2012 la provincia de Cañar es la de mayor incidencia en accidentes de trabajo con 1913.29 casos por cada 100.000 trabajadores, seguida de Napo con 1215,17; en lo que respecta a las principales provincias, Pichincha se sitúa en 371,17 casos y 1037,75 la provincia del Guayas en este mismo año.

Otra de las estadísticas desprendidas del trabajo de Gómez \& Suasnavas (2015), es que el lugar de trabajo es el de mayor índice de incidencia respecto a los initínere y comisión-servicio para ambos períodos de 2011 y 2012. Así mismo, se reporta que los accidentes de trabajo con lesión en los miembros superiores son los de mayor incidencia, 196,4 por cada 100.000 trabajadores, 
seguida por las lesiones en miembros inferiores con 158,8 para el 2012, datos que no varían proporcionalmente en el año anterior.

Con respecto a las consecuencias derivadas por los accidentes de trabajo, Gómez \& Suasnavas (2015), señalan que, las fracturas y luxaciones, torceduras y esguinces, conmociones y traumatismos internos, amputaciones, traumatismos superficiales, contusiones y aplastamientos y otras heridas son las de mayor incidencia. En cuanto a la incidencia de los accidentes de trabajo, esta es muy semejante a lo largo de la semana (lunes a viernes), no existiendo diferencias significativas, siendo de menor incidencia los domingos; así mismo, ocurre con las horas del día en las que con mayor frecuencia se registraron los accidentes, donde las 10:00 h. a.m. es la de mayor incidencia, produciéndose 57,7 accidentes de trabajo a las 10 de la mañana por cada 100.000 trabajadores. Adicionalmente, la incidencia de los accidentes de trabajo que derivan en una incapacidad temporal en 2012 es de 527,02 por cada 100.000 trabajadores.

En el Ecuador según el Ministerio de Relaciones y el Instituto Ecuatoriano de Seguridad Social (IESS) en el año 2015, sólo novecientas (900) empresas de todo el país, han sido evaluadas por medio del sistema Nacional de Gestión de Prevención de Riesgos Laborales de una población total de 179.830 empresas, divididas en 11 sectores.

López (2017), analizan el entorno de trabajo de personal obrero en empresas constructoras y metalmecánicas de la ciudad de Quito - Ecuador, con el fin de entender los riesgos laborales a los que se encuentran expuestos; y, mediante el diseño de perfiles de cargo prevenir accidentes y enfermedades ocupacionales.

Otros aportes como los de Chafla (2017), quien demuestra como el sistema de gestión de seguridad y salud ocupacional, reduce los riesgos laborales en una empresa productora de fertilizantes biológicos y orgánicos en el Chimborazo; Cabrera, Uvidia \& Villacres (2017), quienes implementan un sistema de gestión de seguridad y salud en el trabajo en una empresa de vialidad en la Provincia de Imbabura; Luna, et al. (2017), quienes plantean aspectos legales y técnicos para diseñar un sistema de gestión de seguridad y salud en el trabajo para universidades ecuatorianas; Heredia, Benítez \& Marcillo (2017), quienes realizan un análisis de la normativa vigente en relación a la seguridad y salud ocupacional; Martínez \& Yandún (2017), quienes analizan el alcance de la legislación ecuatoriana en materia de seguridad y salud ocupacional y 
comprueban si los contenidos exigibles en la ISO 26000 están reflejados en la normativa de Ecuador; Ortiz, Gómez \& Vilaret (2018), quienes analizan los accidentes de trabajo ocurridos en una empresa petrolera ecuatoriana entre los años 2014 a 2016; Ulloa-Enríquez (2012), quien comparte una metodología para identificar e incorporar los riesgos de salud y seguridad al sistema de gestión de calidad certificado bajo la Norma ISO 9001:2008, en los procesos de fabricación de embutidos cárnicos de una empresa ubicada en Latacunga, Ecuador; son una evidencia de que en el Ecuador se está trabajando en materia de seguridad y salud laboral.

Se destaca el trabajo de Gómez-García \& Merino-Salazar (2017), quienes realizan un análisis bibliométrico de la producción científica sobre salud laboral publicada por los países pertenecientes a la Comunidad Andina de Naciones en revistas indexadas en la base de datos SCOPUS desde 1995 a 2016, cuyos resultados demuestran la escasa producción científica en esta área de estudio a nivel mundial. Respecto a los países andinos y por orden del número de artículos originales publicados, destaca en primer lugar Colombia con 118 (54,1\%) artículos, Perú con 36 (16,5\%), Venezuela con 30 (13,8\%), Ecuador con 28 (12,8\%) y, en último lugar, Bolivia con $6(2,8 \%)$ artículos sobre salud laboral.

Gómez-García \& Merino-Salazar (2017), consideran que este fenómeno puede ser debido a la inexistente cultura investigativa y deficiente formación desde el pregrado en temas de investigación, así como, la insuficiente importancia de los investigadores de estos países sobre salud laboral. Agregan que, también podría ser debido a la insuficiencia investigadora y acción tutorial de los directores de tesis (profesores) como las posibles causas, dificultando el avance y conocimiento en esta área de estudio para el país.

En el Ecuador, como lo aseguran Toro, Valencia y Oceguera (2014), la prevención de riesgos laborales dista de ser una política de Estado, ya que no se implementan del todo reformas legales permanentes que permitan las actualización en materia de seguridad y salud ocupacional que demanda la legislación nacional e internacional, y en forma preferente garantizar la eficacia en su aplicación que coadyuve a evitar la consumación de siniestros laborales, y a elevar el nivel de protección de la integridad física, mental y social de los trabadores

Uno de los problemas más graves para la presencia de accidentes de trabajo y enfermedades ocupacionales, es el escaso conocimiento y aplicación de las normas que rigen a la seguridad y 
salud en el país, pues queda demostrado que se trabaja muy poco en la divulgación y asesoría acerca de las normas legales, de los métodos y de las normas técnico científicas de la materia (Toro, Valencia y Oceguera, 2014).

\section{Reflexiones finales}

Los cambios que se presentan en el contexto global, donde se destacan los nuevos espacios de trabajo generados por la tecnología y sus nuevas enfermedades y riesgos, representan desafíos para la gestión de la seguridad y la salud ocupacional.

Aspectos como la evaluación de riesgos, la legislación, y la formación, son deberes prioritarios que deben tomar en cuenta quienes conducen los cuadros de investigación y desarrollo, así como aquellos actores de diseños curriculares.

La disponibilidad de información y datos, así como el creciente interés de todas las partes involucradas en el desarrollo organizacional, ha hecho que el tema de seguridad y salud laboral se haya convertido en un asunto multidisciplinario, y que incluso haya evolucionado de un enfoque correctivo a uno preventivo, considerando el bienestar general de las partes.

Aunque no con la misma presencia e impacto con el que se desarrolla la investigación básica y aplicada en el contexto mundial sobre seguridad y salud ocupacional, en el entorno ecuatoriano existe una representación que debe fortalecerse con lo desarrollado a nivel global.

La escasa producción científica presente en el país, puede tener diferentes causas, pero la que más consenso tiene en los autores es la poca cultura de investigación y formación a nivel universitario, a la mano con la escasa oferta de líneas de investigación en el área de seguridad y salud laboral.

\section{Referencias Bibliográficas}

Arias-Mendoza, C. (2017). Implantación de un sistema de gestión de seguridad y salud en el trabajo basado en el modelo Ecuador. Dominio de las ciencias, 3 (4), 264-283. DOI: https://doi.org/10.23857/dom.cien.pocaip.2017.3.4.oct.264-283

Constitución de la República del Ecuador (2008). Registro Oficial 449 del 20-10-2008. San Francisco de Quito. Ecuador 
Cabrera, M.; Uvidia, G. \& Villacres, E. (2017). Sistema de gestión de seguridad y salud en el trabajo, para la empresa de vialidad IMBAVIAL E.P. Provincia de Imbabura. Revista Industrial Data, 20 (1), 17-26. DOI: https://doi.org/10.15381/idata.v20i1.13500

Carvajal, G. \& Pellicer, E. (2009). Tendencias en investigación sobre seguridad y salud laboral. Propuesta metodológica aplicada al sector de la construcción. Revista Ingenierías Universidad de Medellín, 8 (15), 63-73. Recuperado de http://www.scielo.org.co/pdf/rium/v8n15/v8n15a06.pdf

Chafla, D. (2017). Sistema de gestión en seguridad y salud ocupacional en la empresa Agrobest S.A. de la comunidad Gatazo Zambrano, provincia de Chimborazo (Tesis de Maestría). Universidad Nacional de Chimborazo, Ecuador. Recuperado de http://dspace.unach.edu.ec/handle/51000/4334

Gómez, A.; Merino, P.; Espinoza, C. \& Cajías, P. (2018). I Encuesta sobre Seguridad y Salud en el Trabajo en Quito: siniestralidad laboral. Podium, 33, 25-34. DOI: http://dx.doi.org/10.31095/podium.2018.33.3

Gómez, A. \& Suasnavas, P. (2015). Incidencia de accidentes de trabajo declarados en Ecuador en el período 2011-2012. Ciencia \& Trabajo, 17 (52). DOI: http://dx.doi.org/10.4067/S071824492015000100010

Gómez-García, A. \& Merino-Salazar, P. (2017). La investigación científica de los estudiantes universitarios en salud laboral: el caso de Ecuador. SCIENTIFICA, 15 (1), 40-41. Recuperado de http://www.revistasbolivianas.org.bo/pdf/rsscem/v15n1/v15n1_a11.pdf

Heredia, P.; Benítez, A. \& Marcillo, J. (2017). Análisis de la normativa de Seguridad y Salud Ocupacional. Revista Publicando, 4 (12), 3-15. Recuperado de https://www.rmlconsultores.com/revista/index.php/crv/article/viewFile/679/pdf_484

Jilchaa, K. \& Kitaw, D. (2016). A literature review on global occupational safety and health practice \& accidents severity. International Journal for Quality Research, 10 (2), 279-310. DOI: https://doi.org/10.18421/IJQR10.02-04

Jilchaa, K. \& Kitaw, D. (2017). Industrial occupational safety and health innovation for sustainable development. Engineering Science and Technology, an International Journal, 20 (1), 372-380. DOI: https://doi.org/10.1016/j.jestch.2016.10.011 
Jiménez, E. (2017). Evaluación financiera del sistema de seguridad y salud ocupacional en la empresa. Actualidad Contable FACES, 20 (34), 102-118. Recuperado de http://www.saber.ula.ve/bitstream/handle/123456789/42867/art5.pdf?sequence=1\&isAllowed=y

Luna, M.; Álvarez, D. \& Soledispa, S. (2017). Aspectos legales y técnicos para diseñar un sistema de gestión de seguridad y salud en el trabajo para universidades ecuatorianas. Compendium, 20 (38). Recuperado de http://www.redalyc.org/articulo.oa?id=88051773004

López, H. (2017). Perfiles de cargos en seguridad y salud ocupacional en empresas constructoras y metalmecánicas de Quito - Ecuador. Economía y negocios, 8 (1), 1-8. DOI: https://doi.org/10.29019/eyn.v8i1.322

Martínez, M. \& Yandún, E. (2017). Seguridad y Salud Ocupacional en Ecuador: Contribución Normativa a la Responsabilidad Social Organizacional. INNOVA Research Journal, 2 (3), 58-68. Recuperado de http://www.journaluidegye.com/magazine/index.php/innova/article/view/135

Ortiz, J.; Gómez, A. \& Vilaret, A. (2018), Accidentalidad en trabajadores del sector petrolero ecuatoriano: análisis temporal desde 2014 a 2016. Revista Magazine de las Ciencias, 3 (1), 37 46. Recuperado de https://revistas.utb.edu.ec/index.php/magazine/article/view/452/339

Rodríguez, E. (2010). Protección de la seguridad y salud de los trabajadores. Una revisión desde la perspectiva global, latinoamericana y venezolana. 2)1), 81-96. Recuperado de http://www.redalyc.org/articulo.oa?id=215016943006

Rodríguez, M. \& Torre, F. (2010). Caracterización de la evolución en investigación sobre aspectos de gestión de prevención y análisis de accidentes laborales. Revista de la Facultad de Ingeniería Universidad Central de Venezuela, 25 (1), 19-24. Recuperado de http://www.scielo.org.ve/scielo.php?script=sci_arttext\&pid=S0798-

$40652010000100002 \& \operatorname{lng}=e s \& n r m=i s o \& t \operatorname{lng}=e s$

Romeral, J. (2012). Gestión de la seguridad y salud laboral, y mejora de las condiciones de trabajo: El modelo español. Boletín mexicano de derecho comparado, 45 (135), 1325-1339. Recuperado de http://www.scielo.org.mx/pdf/bmdc/v45n135/v45n135a12.pdf 
Toro, J.; Valencia, S. y Oceguera, A. (2014). Marco legal e institucional de la seguridad y salud ocupacional en el ecuador. Acta Republicana, política y sociedad, 13 (13), 101-109. Recuperado de http://148.202.18.157/sitios/publicacionesite/pperiod/republicana/pdf/ActaRep13/10.pdf Ulloa-Enríquez. M. (2012). Riesgos del Trabajo en el Sistema de Gestión de Calidad. Revista Ingeniería Industrial, $33 \quad$ (2), 100-111. Recuperado de http://www.redalyc.org/pdf/3604/360433580002.pdf 